\title{
Without Knowledge Visualization? Proposing a Deconstructivist Approach to Metaphor, Meaning and Perception
}

\author{
Stefan Bertschi \\ University of Zurich \\ stefan.bertschi@loginb.com
}

\begin{abstract}
This paper is designed to examine whether metaphor, meaning and perception are sufficiently dealt with in knowledge visualization. This culminates in the following hypothesis: Because knowledge visualization is a process of construction and reconstruction, the only reasonable approach to evaluate its mechanisms and effectiveness has to be deconstructivist. Such an approach is introduced and examined, leading to primary conclusions and an outlook on a theoretical foundation of an upcoming 'scientific discipline'.
\end{abstract}

\section{Introduction}

At the first symposium on knowledge visualization (IV'05) Noah Bubenhofer and I presented a paper on 'linguistic learning' as a new conceptual focus [1]. We examined the metaphorical face of knowledge visualization and applied initial criticism on a missing theoretical foundation. Thereby, the normative structures in language and visualization, their linguistic coinage, came to the fore. Knowledge is constantly being reconstructed through communication and the use of existing structures and patterns. Thus, one does not have to verbalize and visualize knowledge assets every time anew, but one can refer to antecedent constructions.

At IV'05, we came to the conclusion that knowledge visualization "cannot be more than a mirror image of linguistic (or idiomatic) coinage, which in turn is a mirror image of culture and [...] context." [1, p. 388] Hence, to a certain extent we criticised the overhasty and little reflected foundation of a 'discipline' from within. This previous 'constructivist' approach introduced the observation that "language and culture shape metaphors applied in visualization" [2].

After investigating the basic principles of knowledge visualization and exploring the use of metaphors [1], it would seem it is about time to combine these two 'vistas' and to take a 'tertia vista': a first attempt to highlight what would have to be constructed and reconstructed in knowledge visualization. In line with the construction and reconstruction of knowledge, it seems promising to introduce a new access to its visualization. In section 2, I shall introduce a deconstructivist approach.

Knowledge visualization aims at augmenting communication via graphic means that mediate knowledge through metaphors. Following the 'tertia vista' in section 3, I rejoin the debate on metaphor in knowledge visualization in section 4 . In the penultimate section, I argue that semiotization and symbolization are plausible ways to integrate the 'tertia vista' and metaphor: A functional perception is dependent on the intended meaning of visualization. Knowledge visualization needs an underlying theoretical approach before it is "time to establish visualization science as a scientific discipline" [4]. Finally, I present the major conclusions and an outlook that can be drawn from this analysis.

\section{Semiology and deconstruction}

Whereas the previous paper was aimed at giving some thought to a "semiology of the communication of knowledge" [1, p. 388], this paper resumes this train of thought. 'Linguistic learning' already pointed in a direction predetermined by the so-called 'linguistic turn', a paradigmatic turn based on "the view that philosophical problems are problems which may be solved (or dissolved) either by reforming language, or by understanding more about the language we presently use." $[5$, p. 3] These problems particularly refer to the question about the 'conditions of the possibility' of knowledge and have effects not limited to philosophy.

In the linguistic turn, the question 'What is (an) X?' was replaced by 'What is the meaning of ' $X$ '?' (i.e., discovering the nature of ' $X$ ') and subsequently by 'How do we (or should we) use the word ' $X$ '?' (e.g., to improve and sustain the transfer of knowledge) [5, p. 4]. Finally, in discourse linguistics this would lead to the question: What does one have to know in order to 
know (to be aware of) the use of ' $X$ '? Hence, "we cannot investigate [...] the concept of $X$, except by investigating our use of words" [5, p. 12]. The "linguistic turn' questions the idea of language as a transparent medium for the perception and communication of reality. Language is seen as a condition of thinking which cannot be deceived, i.e., all human knowledge is structured by language; reality beyond language is not existent or at least unattainable [6, p. 371].

The problem of language as a medium and a carrier of meaning is reflected in 'semiology', the study of the function of signs and symbols in communication [13, p. 95 f.; cf. 3, p. 34]. It is suspected that knowledge visualization is effected by this problem. Therefore, a convergence seems fruitful: Both try to describe 'reality'; both engage in linguistic reference, "the nonverbal 'outside' to which language refers, by which it is conditioned and upon which it acts." [7, p. 27] The following definition sheds new light on this particular reference: "Semiology, as opposed to semantics, is the science or study of signs as signifiers; it does not ask what words mean but how they mean." [7, p. 28, my italics] In his definition, Paul de Man conceives 'semiology' rather as a kind of deconstructive reading. Applied to the present context, the interest in knowledge visualization does not merely lay on what is said but on how (and additionally why) it is said and how and why visualizations mean something.

In a critical view of knowledge visualization, one may assume that its 'problem determination' and 'problem solving' are situated on the side of content and not reflection (theory). The present approach does not raise the question what visual representations mean but how and why they mean. Therefore, "[ $[\mathrm{t}]$ he demystifying power of semiology" seems to be a suitable approach because it "explodes the myth of semantic correspondence between sign and referent" [7, p. 28]. It only remains what is called 'deconstruction'.

In order to understand the idea a sign conveys, it is to be interpreted. This is necessary because "the sign is not the thing but a meaning derived from the thing by a process here called representation"; " $[\mathrm{t}]$ he interpretation of the sign is not [...] a meaning but [reference to] another sign" [7, p. 29]. In this poststructuralist paradigm conjoins the recourse to the linguistic turn $[6, \mathrm{p}$. 523]. Poststructuralism develops its specific theories out of a rigorous semiotization of the world. Thereby, deconstruction is a "subversive principle of the approach to texts "from within" [6, p. 101]; deconstruction is theory and practice of poststructuralist philosophy and literary criticism. Its principle is mindful of criticising the little reflected foundation of knowledge visualization 'from within'.

Broadly defined, deconstruction is a type of reading texts, of revealing the underlying circumstances and contexts in which they are written and received. The deconstruction of a text proceeds "by the careful teasing out of warring forces of signification within the text itself. [...] [It] is a reading which analyzes the specificity of a text's critical difference from itself." [8, p. 3] Thereby, deconstruction investigates "the tension between modes of signification, as between the performative and constative dimensions of language." [9, p. 122] As a result, every deconstructivist approach can be seen as some kind of depiction or visualization, and every visualization should be seen from such a perspective.

\section{Knowledge visualization: tertia vista}

The "new research focus" (or "field" or "area" [cf. 3 , p. 14]) of knowledge visualization aims at depicting "how complementary visual representations can be used to improve the transfer of knowledge in organizations." [3, p. 2] Examples of representations are images, objects, interactive visualizations or stories. The following hypothesis emanated from the 'prima vista' applied in [1, p. 384]: Knowledge visualizations "aim particularly at portraying the reconstruction of patterns". In the following, we take a 'tertia vista' by analyzing how these patterns are being reconstructed.

One important aspect is noted right at the beginning of the first, major scholarly work on knowledge visualization: "In contrast to information, which is explicit, knowledge has to be re-constructed by each individual. This process happens through communication and interaction with explicit information - verbal or visual." [3, p. 12, my italics] In his thesis, Remo Burkhard states "the main need [...] is to create a theoretical basis for the new research field Knowledge Visualization." [3, p. 15, my italics] Considering its parent disciplines, he senses a "need for the transfer of insights" in information visualization [3, p. 16] and a "need to care for the recipient" in visual communication $[3, \mathrm{p}$. 17]. It should be noted that knowledge visualization yet seems to be closer to 'visual communication' than to 'information visualization'. Because "there is a need to complement verbal representations with visual representations", novel methods are seen in "the use of visual metaphors to transfer and remember complex concepts, or visual storytelling to disseminate knowledge." $[3$, p. 17] However, to integrate the perspective of the recipient is important for both (or all three) disciplines, but it is not sufficiently leveraged in any of them.

A main observation of constructivism is that "knowledge is actively constructed or re-constructed by each individual. And individuals need to accord meaning to knowledge instead of just accumulating facts." [3, p. 25] Furthermore, "collaboration is important for social negotiation on meaning, for the testing 
of ideas, and the exchange and discussion of different perspectives." However, everyone who wants to "transfer knowledge to one or more individuals, with similar or different backgrounds" [3, p. 31] has to be aware that "visualizations need to be customized $[. .$. in a way that the recipient can re-construct knowledge as intended by the sender" [3, p. 54]. To allow this reconstruction, 'knowledge communication' should not only transfer facts, to answer questions like 'what?' but transfer insights, to answer questions like 'why?' and 'how?' The latter questions lead on to perception as "a set of processes by which we recognize, organize, and make sense of stimuli in our environment." [3, p. 40] Because of its focus on content rather than theory, these aspects of constructivism and perception are only insufficiently addressed in knowledge visualization.

Furthermore, Burkhard aims "to make new contributions by establishing the new field Knowledge Visualization in the context of business knowledge management." [3, p. 52] This focus is too restrictive, as there is more potential in the construction and reconstruction of knowledge or so-called 'knowledge visualization'. Burkhard's contributions include other aspects, like the risks of visualization, e.g., "reality could be distorted through [misinformation and] misinterpretations" or merely by over-simplification [3, p. 116]. However, it is not intended to address each of them in isolation. Instead I rejoin and extend the previous debate on metaphors.

\section{The concept of metaphor: a rejoinder}

In his thesis [3], Burkhard uses the term 'metaphor' 62 times, but he does not sufficiently explain what is meant, let alone tailor this figure of speech to knowledge visualization. For instance, in order to introduce the term 'metaphor' [3, p. 62] he refers to yet another incomplete explanation of this particular figure of speech in knowledge visualization [cf. 18]. Burkhard introduces the so-called 'tube map' metaphor as the most prominent example [3, pp. 79-84], but does not leverage its potential of being used as a 'case study' to illustrate the basic functionality of metaphor in knowledge visualization. Another example would be the reference to 'mental images' and symbolism [3, pp. 105-107] without any further explanation of these concepts relevant to the use of metaphors.

In his discussion of the cognitive benefits of visual representations, Burkhard states: "Visual imagery $[. .$. suggests that visual recall is more efficient than verbal recall. $[. .].[\mathrm{I}] \mathrm{t}$ is clear that humans have a natural ability to use images" because they "think in images, and accordingly, can more easily remember by the use of images." [3, p. 104] Apart from mentioning the benefits for recall and understanding of information, this important and legitimating aspect for visualization is not further elaborated, let alone sufficiently connected to metaphor.

It is fair to say that some basic concepts are mentioned in knowledge visualization [cf. 3] and that the use of 'visual metaphors' is prominent [cf. 18]. However, a solid introduction and critical discussion of 'metaphor' is missing to this day. I shall not elaborate in further detail on this, but instead refer to the criticism brought forward in [1, p. 386].

\subsection{What is a metaphor?}

Common definitions of metaphor are: "metaphor is that figure of speech whereby we speak about one thing in terms which are seen to be suggestive of another" [10, p. 15, my italics], and "metaphor is seeing something from the viewpoint of something else" [11, p. 170, my italics]. Although reflecting Aristotle's definition of metaphor [cf. 12, p. 4], each takes a different perspective (i.e., sender versus recipient). According to Merriam-Webster, a metaphor is "a figure of speech in which a word or phrase literally denoting one kind of object or idea is used in place of another to suggest a likeness or analogy between them". Compared to the previous definitions, the latter is mediating between sender and recipient and focuses on the act of using metaphors.

Only since the mid-18th century and Giambattista Vico's analyses, the view appeared "that metaphor characterizes human thought and language in a truly fundamental way" $[12$, p. 3]. In the early 1980s, it was pointed to the impossibility of non-metaphorical thought because metaphoricity of language is total [1, p. 384]. In the early 1990s, scholars began "investigating the possibility that metaphor is not only a form of speech, but more fundamentally a form of thought", playing "a fundamental role in the acquisition and extension of knowledge" [12, pp. 1, 3]. In this regard, it could be stated that "[a]ll knowledge is ultimately rooted in metaphorical (or analogical) modes of perception and thought." [12, p. 2] Today, the view that human categories of understanding are metaphorical in nature and function is widely accepted: "Through a metaphor, one is not simply conveying facts but enriches the communication with imagery. A metaphor stimulates the mind to imagine." [13, p. 92] Of foremost importance is the "conception of metaphor as the fountain of meaning and truth" [14], playing a foundational role in human understanding. It was, for instance, Friedrich Nietzsche who claimed that the metaphor plays a central role in the way we make sense of the world. By "providing a way of imposing or discovering structure" [14], metaphors reveal how our language is related to the world. 


\subsection{The problem of metaphor in knowledge visualization}

It is essential to understand "how a metaphorical use or interpretation of language can convey a kind of information, or bear cognitive significance, above and beyond what we might all agree is what it says" $[15, \mathrm{p}$. 188]. The following quote depicts the problematic characteristic of this setting: "The information or knowledge or cognitive content [...] communicated by a metaphor is at least in part a function of the specifically metaphorical mode by which the utterance is interpreted." [15, p. 192] First, the quote points to an uncertainty in terminology; shall we say information or knowledge or cognitive content? Second, metaphors point to the strong interpretational interrelation of intention and perception. Third, the quote points to the communicational ability and capability, i.e., to communication as the lowest common denominator. As already outlined in [1], "metaphorical interpretations are highly dependent on and sensitive to their contexts of utterance." [15, p. 194]

Additionally, metaphors "admit endless or at least open-ended interpretations; what they mean or communicate is never antecedently fixed." [15, p. 205] This certainly leads to problems because it is difficult to grasp "the context in which we are supposed to interpret the metaphor." $[15$, p. 207] For instance, this can be due to remote or culturally distant contexts not sufficiently discovered or a multiplicity of possible sets of presuppositions, making it difficult to know the relevant presupposition.

Regarding its metaphoricity, it was contended that pictorial and linguistic visualization is one and the same [1, p. 384]; they represent two sides of the same coin as they are complementary parts of communication [3, p. 103]. Eventually, there is a significant difference between pictures (i.e., depictive presentations) and linguistic descriptions: "presentations display rather than describe the information, or content, they convey." [15, p. 200] In short: "A picture is not worth a thousand words, or any other number. Words are the wrong currency to exchange for a picture" [Davidson in 15 , p. 220]. This leads to the following questions: Which meaning do people apply to what they 'see' and discover? What are they told to 'see'? How is intersubjective meaning possible? Does knowledge visualization merely denote a necessity for the metaphorical mapping of knowledge?

This metaphorical character is obvious because knowledge visualization "works with an unobtrusive 'pie chart' or a more complex 'tube map' which can be exhausted of its metaphoricity" [1, p. 384]. It was noticed that "the medium of knowledge visualization is in most cases a metaphor" [1, p. 385], but even metaphors cannot ensure that meaning is transferred identically to all recipients. A possible answer would be to choose "the most promising [visualization] type to transfer knowledge to the specific stakeholder" and to ensure effective communication [3, p. 112]. However, this multiplicity of 'dependent perceptional offers' is pointing to knowledge visualization's shortcomings in addressing metaphors. The fact that "knowledge must be recreated in the mind of the receiver, which depends on the recipient's cognitive capacity to process the incoming stimuli" [3, p. 113] and which is not yet sufficiently reflected on, is pointing in the same direction.

There is other evidence to be found at the intersection of metaphor and picture. The idea, metaphors are like pictures, highlights "a pictorial dimension that distinguishes the character of a metaphor from that of non-metaphorical expressions." [15, p. 220] Therefore, "[w]hat makes a metaphor pictorial [...] lies in its mode of symbolization", i.e., "the system or schema in which it functions is [...] not finitely differentiated" $[15$, p. 221 , my italics]. Because the cognitive significance of a metaphor depends on its schema, and metaphorical interpretation is "non-finite", knowledge visualization is affected and lacks "the means to express that knowledge in a finitely differentiated symbol scheme" [15, p. 222, my italics]. This calls for a deconstructivist approach and for finding a solution to this dilemma.

\section{The social meaning of perception}

Not only business knowledge management, the self-appointed focus of knowledge visualization, depends on awareness, perception and the recognition of meaning. How is something done and why? Is it able to fulfil its objectives? These questions apply to most aspects of human life. In this particular context the meaning (and 'meaning-making') of knowledge visualization would ask for an extensive sociological reconsideration. To constitute a meaningful practice within the transfer of knowledge, a pursuit of meaning seems inevitable. The constructivist approach introduced in [1] was mainly about social construction: "People think in pictures, so knowledge must be recreated in the mind of the receiver." [2] We made clear that " $[\mathrm{m}]$ odes of thought can be adequately understood if their social origins are familiar and the pictures are defined by language used in a specific culture at a specific time." [2; cf. 1, p. 385]

A relevant general finding of 'cognitive constructivism' is "that subjects actively construct their experience." $[16$, p. 6] It is crucial to acknowledge and theoretically incorporate that the receiver is not merely a passive recipient of environmental information but participates in the structuring of external reality. In 
review, this "implies the semiotization of meaning, that is, its revision as a complex subjective construction [...] which is permanently modified in communicative use." [16, p. 20] Knowledge visualization demonstrates a slight awareness for the interpretational process of meaning: "People acquire knowledge in a complex process where prior experience and several other factors have major influences on the process of constructing meaning." [3, p. 121, my italics] Because knowledge is a socially mediated product, perception demands our undivided attention. The deconstructivist approach is used to see meaning as a social construction, and the sociology of knowledge analyzes the social construction of reality, again leading to the necessity to include sociological reflection in knowledge visualization. It is rather important to understand the subjective meaning of people's experiences [cf. 1, p. 385], especially for a research area which has 'meaning' as its main currency.

Social construction is 'meaning production' and relates to the "symbolic structuring of meaning and the generation of symbolic orders" [17, p. 2]. This symbolic ordering emerges from social processes of interaction and practices of representation (mainly through language use), and is closely connected to metaphor because "the formal representation of social reality, as with other realities, is through and through metaphoric." [11, p. 170] Hence, it is necessary to study the practices by which we 'construct' and perceive the world (i.e., our conception of reality) and facilitate these insights to assess the basis of knowledge visualization and to build sound theoretical concepts. A deconstructivist approach - building on a 'tertia vista' and the debate on 'metaphor' - is offered as a starting point for such an endeavour. Once again, the point is not what is constructed and reconstructed, but how (and why) these meanings are constructed and reconstructed.

\section{Conclusion}

Even though I followed a critical approach, it leads to the conclusion that there should not be a 'without knowledge visualization'. In contrary, this research area not only has the potential to solve "the predominant problems in organizations", i.e., to communicate complex contents to diverse audiences [3, p. 131], but to pose important problems related to this communication. Knowledge visualization "aims to improve the transfer and the creation of knowledge" by giving people "richer means to express what they know" [3, p. $135]$, but the research area would need an improved theoretical foundation of metaphor, meaning and perception to be able to do so.
To mention the importance of the recipient $[3, \mathrm{pp}$. $17,54,135]$ is not sufficient as long as there is no sound theoretical basis to include this aspect. It is fair to say that a more thorough analysis of the use of metaphor, meaning and perception in knowledge visualization and its contribution would be appropriate. Considering the foundation of knowledge visualization as a 'scientific discipline', building a "big picture and mutual agendas" [4, p.189], this seems more than necessary. There is another reason for this necessity; the assumption that visualizations will lose their character as static objects in a familiar sender-recipient setting and foster to "establish an iterative, collaborative process where the visualization (and new knowledge) is dynamically co-created." [3, p. 140] A deconstructivist approach may facilitate the design as well as the evaluation of knowledge visualization tasks. In this paper I presented first steps toward such an approach.

The main research questions, originated in this paper but left open, concern; first, from a 'linguistic perspective', the supposition of the interrelation of narrative and story(telling) in metaphors and beyond, second, from a '(de)constructivist perspective', the specification of the 'meaning of meaning' (i.e., 'meaning-making', and the 'understanding of understanding' including social and cultural knowledge), third, from a 'social perspective', the power of ontology and the primacy of representation as an explanatory concept, and fourth, the supposition of the necessary connection between these three concepts, ranging from concrete to abstract levels or more generally: the perception of visualized reality shaped by the dichotomy of subjectivity and objectivity.

Finally, I conclude that metaphor, meaning and perception are not sufficiently elaborated in knowledge visualization. To provide necessary enhancements these would need to be composed in an ampler scholarly format. The preliminary findings indicate that it makes sense to visualize knowledge, but the question remains whether it is the time to establish such a research area as a 'scientific discipline'. The answer would be: certainly not as long as the deficits mentioned prevail.

\section{Acknowledgements}

I should like to thank Noah Bubenhofer and the three anonymous reviewers for valuable comments on earlier drafts of this paper.

\section{References}

[1] Stefan Bertschi and Noah Bubenhofer. Linguistic Learning: A New Conceptual Focus in Knowledge Visualization. In Ninth International Conference on In- 
formation Visualisation (IV'05). London. July 2005. IEEE Computer Society Press. 383-389.

[2] Anna Ursyn and Ebad Banissi. Information Visualization (IV): Notes about the 9th IV'05 International Conference, London, England. In Body, Space \& Technology 6. 2006. http://people.brunel.ac.uk/bst/vol06/ annaursyn/ (Retrieved on January 5, 2007).

[3] Remo Burkhard. Knowledge Visualization: The Use of Complementary Visual Representations for the Transfer of Knowledge - A Model, a Framework, and Four New Approaches. D.Sc. thesis. Swiss Federal Institute of Technology (ETH Zurich). 2005.

[4] Remo Burkhard. Is it Now Time to Establish Visualization Science as a Scientific Discipline? In Tenth International Conference on Information Visualisation (IV'06). London. July 2006. IEEE Computer Society Press. 189-194.

[5] Richard Rorty. Metaphilosophical Difficulties of Linguistic Philosophy. In The Linguistic Turn: Recent Essays in Philosophical Method. Richard Rorty, Ed. Chicago, University of Chicago Press. 1967. 1-39.

[6] Ansgar Nünning, Ed. Metzler Lexikon Literatur- und Kulturtheorie. 2nd ed. Stuttgart, Metzler. 2001.

[7] Paul de Man. Semiology and Rhetoric. In Diacritics 3 (3). 1973. 27-33.

[8] Barbara Johnson. The Critical Difference. In Diacritics 8 (2). 1978. 2-9.

[9] Jonathan Culler. Literary Theory: A Very Short Introduction. Oxford, Oxford University Press. 1997.
[10] Janet Martin Soskice. Metaphor and Religious Language. Oxford, Clarendon Press. 1985.

[11] Richard H. Brown. Social Theory as Metaphor: On the Logic of Discovery for the Sciences of Conduct. In Theory and Society 3 (2). 1976. 169-197.

[12] David E. Leary. Psyche's Muse: The Role of Metaphor in the History of Psychology. In Metaphors in the History of Psychology. David E. Leary, Ed. Cambridge, Cambridge University Press. 1990. 1-78.

[13] Jason Maratos. The Power of Myth as Metaphor. In Group Analysis 39 (1). 2006. 87-99.

[14] William Grey. Metaphor and Meaning. In Minerva: An Internet Journal of Philosophy 4. 2000. http://www.ul. ie/ philos/vol4/metaphor.html (Retrieved on January 4, 2007).

[15] Josef Stern. Knowledge by Metaphor. In Midwest Studies in Philosophy 25. 2001. 187-226.

[16] Carlos Cornejo. Who Says What the Words Say? The Problem of Linguistic Meaning in Psychology. In Theory \& Psychology 14 (1). 2004. 5-28.

[17] Reiner Keller. Analysing Discourse: An Approach from the Sociology of Knowledge. In Forum: Qualitative Social Research 6 (3). 2005. http://www.qualitative-research.net/fqs-texte/3-05/053-32-e.pdf (Retrieved on January 13, 2007).

[18] Martin J. Eppler. The Image of Insight: The Use of Visual Metaphors in the Communication of Knowledge. In Journal of Universal Computer Science: Proceedings of I-KNOW'03. Graz. July 2003. 81-88. 